\title{
Bus Identification Device for Blind People using Arduino
}

\author{
K. Arun Kumar ${ }^{1}$, P. Sreekanth ${ }^{2}$ and P. Rajashekar Reddy ${ }^{3}$ \\ ${ }^{1}$ Asst. Professor, CVR College of Engineering/ECE Department, Hyderabad, India \\ Email: arun.katkoori@gmail.com \\ ${ }^{2}$ Asst. Professor, CVR College of Engineering/ECE Department, Hyderabad, India \\ Email: Sreekanth.isoft@gmail.com \\ ${ }^{3}$ Asst. Professor, CVR College of Engineering/ECE Department, Hyderabad, India \\ Email: raju.sheker@gmail.com
}

\begin{abstract}
Generally, journey in bus is a safe and comfort factor, but due to increase in number of buses and passengers it's going to be tougher now-a-days and it will be even more difficult for blind people to travel in bus. This paper presents a low cost and easy to build and use system for blind people to identify which bus is approaching the bus stop. The main purpose is to provide a bus detection system for blind people by assigning different tags to different buses with the help of Radio Frequency Identification (RFID) technology. In the experiment set-up, Arduino, RFID reader and tags, bluetooth module for voice announcement and Mobile phone with Serial Monitor App which gives voice commands are used. Whenever the bus comes to the stop, the RFID reader will be reading the tag (which is given to every bus for particular route) depending upon the tag it will give voice intimation so that blind people can know the bus number and related information about bus.
\end{abstract}

Index Terms: Bus identification device, Blind people, RFID, Bus, Bus station, Voice message.

\section{INTRODUCTION}

Today, one of the most worldwide occupation is helping and supporting visually impaired person. In most physical environments, the visually impaired have difficulty in accessing information about transport, stops, terminals, vehicles, schedules, maps and directories which prevent them from using public transport effectively. Hence, there is a need to make their lives more comfortable by introducing a system that helps them enjoy transportation services independently and freely like ordinary people, without relying on others. [5] This paper focuses on a bus identification system using Radio Frequency Identification (RFID) technology. The RFID system uses tags, through which information embedded on the tags are read by RFID readers. The proposed system eliminates the need for help on whom the blind people relies for guidance to board the required bus to reach destination. [6] The problems that surfaced during the project were selecting the right kind of device and interfacing these devices appropriately. The approach used was to minimize costs and complexity.

Over the years, there have been comparable research endeavors to create implanted gadgets to mitigate issues. The Talking Signs distinguishing proof framework [6] comprises of infrared (IR) transmitters fused in the target boards of transports that transmit route data. This method is difficult because, an IR beam is exceptionally directional, the person must point the receiver towards the transmitter on the bus. Exact bus location is unknown for the blind person.

Broadcasting bus- is a GPS based framework reporting the bus number upon arrival at the bus stop. These smart bus stops are not satisfactory when outfitted just with the voice detailing framework since voice data is hard to interpret when numerous buses simultaneously arrive at the similar terminal. [3]

\section{Proposed Method}

This system is designed to provide information for blind assisting with voice command signals utilizes RFID technology for task of identification. [2] In this system, a RFID reader is installed in every bus near driver which is integrated with Arduino Uno with bluetooth module. Voice assistance equipment like loud speaker is installed in every bus stop. Also, a mobile phone is used with an applicationSerial Monitor, which will give voice commands. The block diagram is shown in following figure 1.

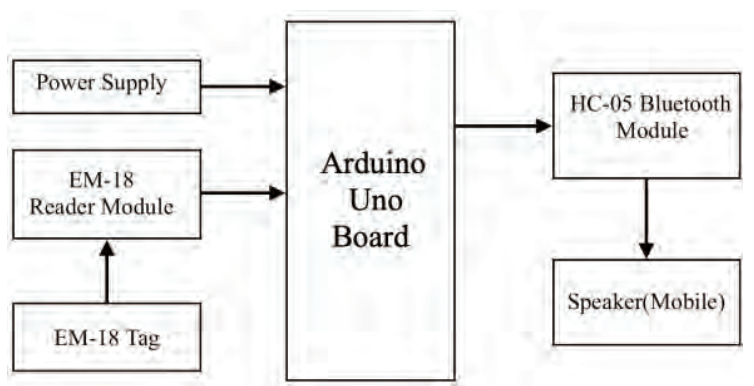

Figure 1. Block Diagram of Bus Identification Device for blind people using Arduino

The key steps of this implementation are

- Placing the RFID tag in front of RFID reader. 
- RFID reader provides a signal to Arduino Uno about the detection of RFID tag which contains information about the bus.

- Then, Arduino Uno sends signal to Bluetooth module which is connected to mobile(here) with bluetooth.

- The application will produce a voice message.

\section{A. Methodology and Working}

The bus drivers are equipped with a unique RFID tag. When a bus approaches a bus stop, the driver will be place the RFID tag given to that person, near the RFID reader. RFID reader receives the information of the bus through a unique identification code. [8] This reader also detects RFID tag which consists of information related to the bus like bus number and places. The RFID reader acts as a transmitter and transmits to Arduino, [1] the identification code of the bus and with the program dumped in Arduino with the unique identification code the Arduino will be send signals to the bluetooth module.

Now, Bluetooth module acts as receiver and receives the signals from Arduino. [1] The voice-based play back system is already fed the information and is transmitted out through speaker.

\section{B. Hardware Resources}

The main hardware resources in this implementation are Arduino Board, Bluetooth module, RFID reader and tags.

\section{i) ARDUINO UNO}

The Arduino is a group of smaller scale controller sheets to disentangle hardware plan, testing and prototyping for craftsmen, programmers, and numerous experts. It can be used it as brain for robots, to assemble new advanced music instruments, or to manufacture a framework that lets home plants tweet once they're dry. Arduinos (Arduino Uno is utilized) are worked around an ATMEGA microcontrollerbasically a total PC with all the components on a solitary chip. Unlike, say, a Raspberry Pi, it's designed to attach all kinds of sensors, LEDs, small motors and speakers, servos, etc. directly to these pins, which can read in or output digital or analog voltages between 0 and 5 volts. Figure 2 demonstrates the Arduino Uno board. Using USB, the Arduino board is associated with the PC. The program is written in a basic language like $\mathrm{C}, \mathrm{C}++$, or Java on Arduino IDE by transferring accumulated code to the board. [1] The Arduino can keep run with the USB connected back to the computer, or remain solitary without it. No console or screen required, simply power when the code is modified.
The Arduino Uno can be powered via the USB connection or with an external power supply. The board can operate on an external supply of 6 to 20 volts. If supplied with less than $7 \mathrm{~V}$, however, the $5 \mathrm{~V}$ pin may supply less than five volts and the board may be unstable. If using more than $12 \mathrm{~V}$, the voltage regulator may overheat and damage the board. The recommended range is 7 to 12 volts.

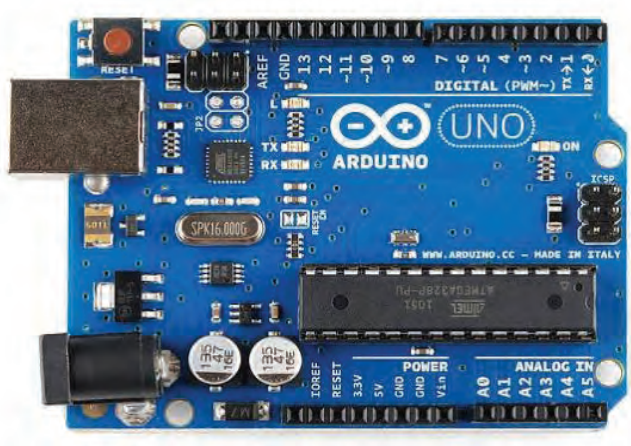

Figure 2. Arduino Uno Board

\section{ii) BLUETOOTH DEVICE}

HC-05 module is used as a Bluetooth Serial Port ProtocolSPP module. It is designed for transparent remote serial connection setup. It can be utilized as master or slave arrangement. The features of this module-

- $\quad$ Bluetooth V2.0+EDR.

- 3 Mbps Modulation with complete $2.4 \mathrm{GHz}$

- CSR Blue center 04-External single chip Bluetooth framework with CMOS innovation and with AFH- Adaptive Frequency Hopping feature.

By default, the module is used as slave. The role of the module (Master or slave) can be designed just by AT directions. The HC-05 is a cool module which can include full duplex remote usefulness. It can be used between two Arduinos to communicate with each other.

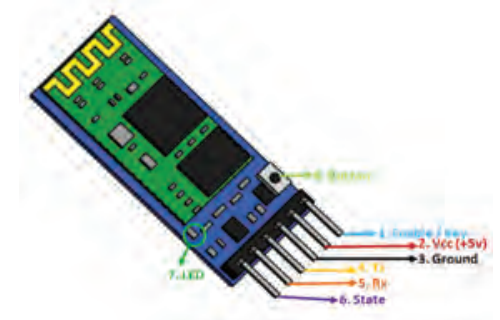

Figure 3. Bluetooth module-HC 05

HC-05 operated in modes- i) Data mode, ii) AT command mode. In data mode, data is transmitted and received from one Bluetooth device to another. In second mode, the default device settings are changed. By using pin number 1 (i.e. Enable/Key), it can be operated in any one the two modes. 
This module operated using SPP protocol, it is easy to pair with microcontrollers. Connect the Rx pin (pin number 5) to the Tx pin of microcontroller, Tx pin (pin number 4) to the $\mathrm{Rx}$ pin of microcontroller and power the module with 5 volts.

\section{iii) RFID READER AND TAGS EM-18}

RFID is used to characterize the system by sending a unique serial number of an object wirelessly using RF waves.

RFID empowers distinguishing proof from a separation and dissimilar to prior standardized tag innovation, it does so without requiring a viewable pathway. RFID labels bolster a bigger arrangement of one of the kind IDs than standardized identifications and can fuse extra information, for example, maker, [4] item type and even measure natural factors, for example, temperature.

EM18 module emanates out $125 \mathrm{KHz}$ through the curls. At the point when a $125 \mathrm{KHz}$ RFID latent tag is purchased to the field module will get empowered from the field. By the adjustment in balance current through the loops, the label will send the data back to the program memory exhibit.

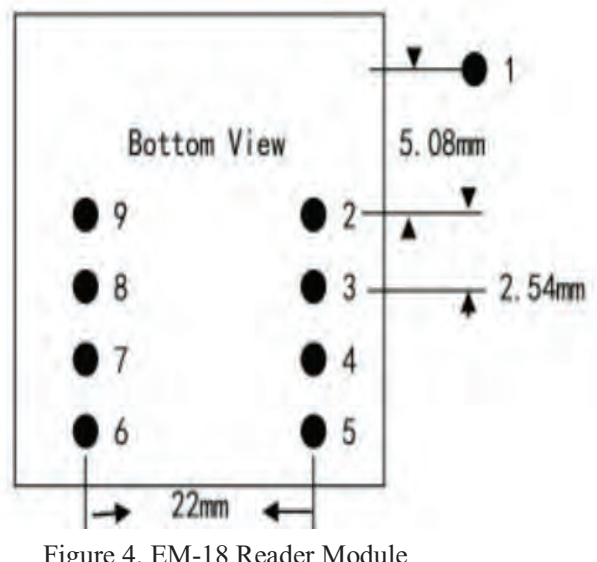

Figure 4. EM-18 Reader Module

Data is stored in any one of two types of memory. One is ROM- read only memory another one is RAM-random access memory. In ROM, once the data is placed, it cannot be modified whereas in RAM, data can be changed. RAM is also called Read/Write Memory.

Connections among the above hardware resources is shown in figure 5 .

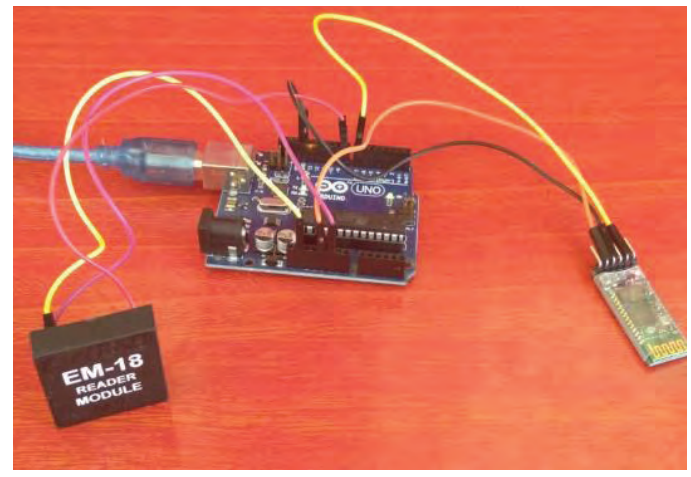

Figure 5. Arduino Board connections to Bluetooth and RFID reader

\section{Arduino Software}

The IDE of Arduino makes it simple to compose code and transfer it to the different boards. It works on Windows, Mac OS X, and Linux. Java is used in the environment and dependent on processing and another open-source programming. This product can be utilized with any Arduino board. Arduino is an open-source prototyping stage dependent on simple to utilize equipment and programming. [1] Arduino can peruse inputs- light on sensor, a finger on a catch, or a message- and transform it into a yield- initiating an engine, turning on a LED, distributing something on the web and some more. To do as such utilize the Arduino programming language (in the view of wiring), and the Arduino software (IDE), based on processing.

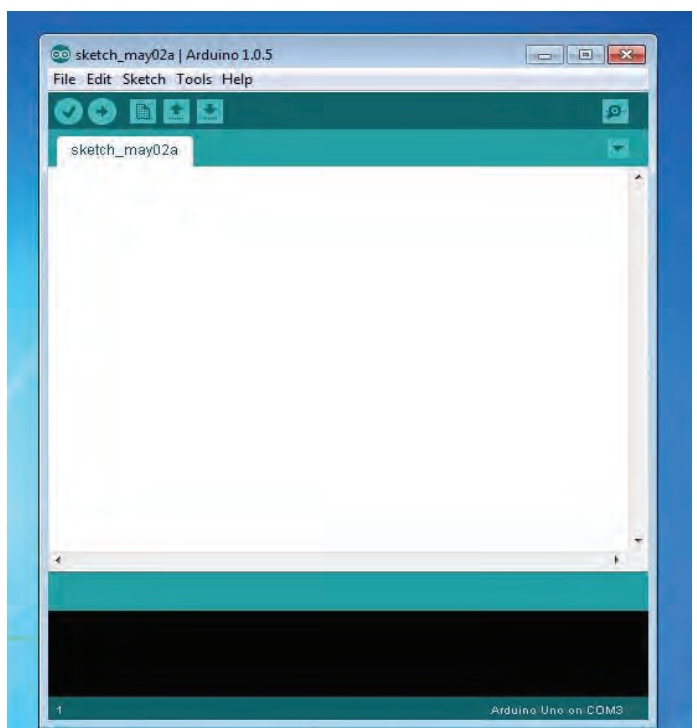

Figure 6. Arduino IDE 


\section{Features of Arduino IDE}

Inexpensive: Arduino boards are relatively inexpensive compared to other microcontroller platforms.

Cross-platform: The Arduino Software (IDE) runs on Windows, Macintosh OSX, and Linux operating systems. Most microcontroller systems are limited to Windows.

Simple, clear programming environment : The Arduino Software (IDE) is easy-to-use for beginners, yet flexible enough for advanced users to take advantage of it as well.

Open source and extensible hardware/ software: The Arduino boards have common license, so experienced circuit designers can make their own version of the module, extending it and modifying it.

It is an open source and libraries are written in $\mathrm{C}++$.

\section{Flow Chart}

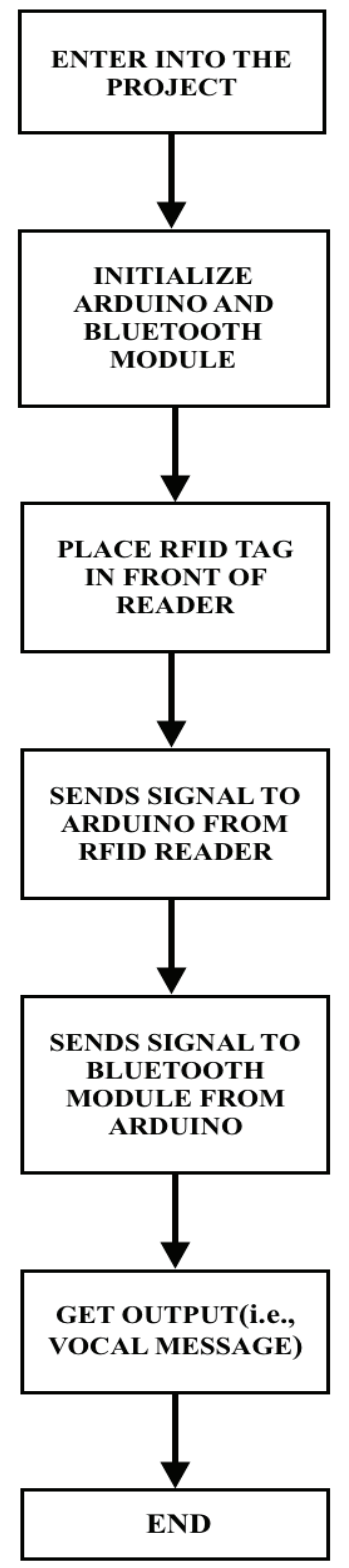

Figure 7. Flow chart of Bus Identification Device for blind people using Arduino

\section{RESUltS}

The below figures show the output after placing the card for five times. Each time by placing the card, the output gets in both Arduino serial monitor, and serial monitor app related to the information stored about the bus. By placing the card again i.e. $6^{\text {th }}$ time, the information will be given in reverse order. When the bus starts again from the end location, now it becomes the starting location.

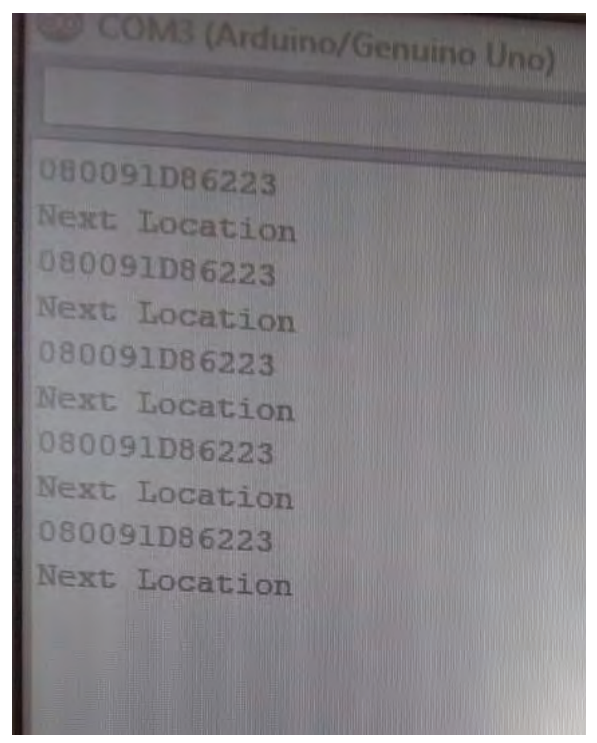

Figure 8. Output after placing the card for five times on Arduino serial monitor

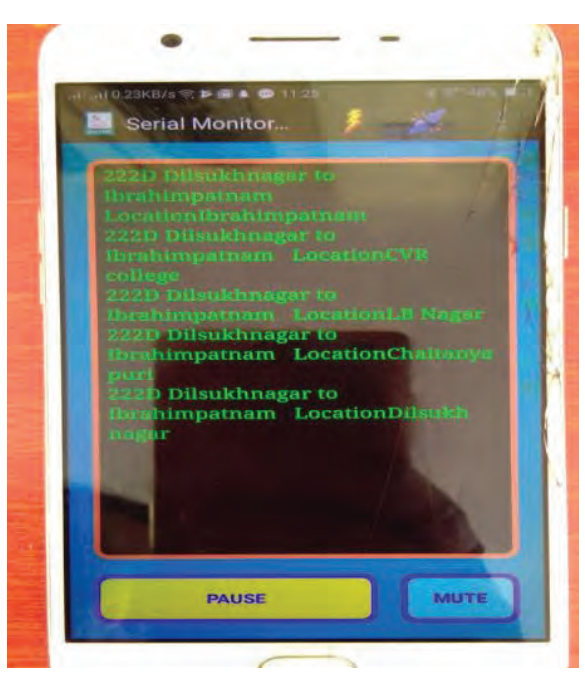

Figure 9. Output after placing the card for five times on Mobile app

When placed the card for sixth time, the ending location now became the starting location and the journey becomes reverse. 


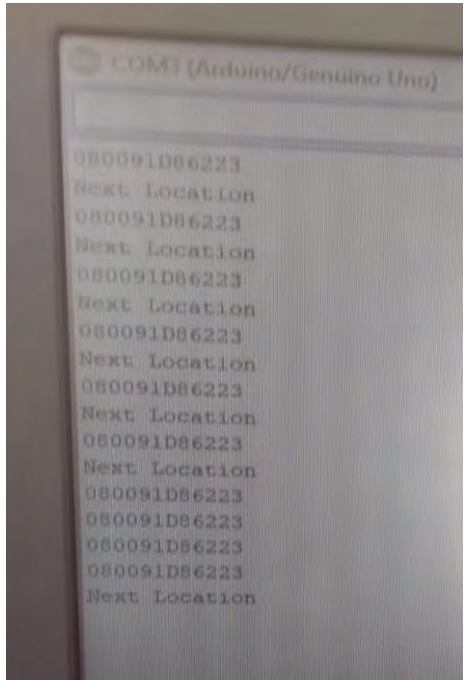

Figure 10. Output after placing the card for five times on Arduino serial monitor

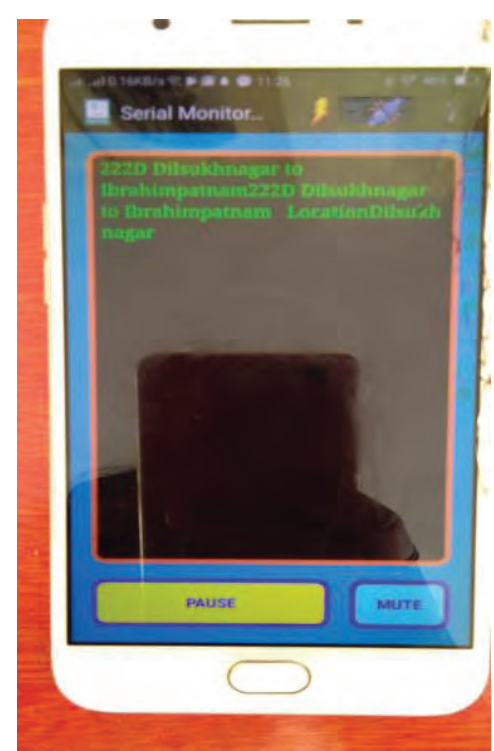

Figure 11. Output when Placed for Sixth Time on Mobile app

\section{CONCLUSIONS}

In this paper, the bus identification system for blind people using RFID was successfully implemented. The proposed technique is more suitable for blind passengers. When the blind passenger reaches the bus station, with the help of voice synthesizer can find the buses that pass through a location.

\section{REFERENCES}

[1] Arduino for Beginners: Step-by-Step Guide to Arduino (Arduino Hardware \& Software) By Simon Knight.

[2] B.N Kiran, Smitha B.C,Sushma K.N, Varsha R.Gowda,'Impl ementation of RFID for blind bus boarding system
,'International Journal of Science Engineering and Applied Science(IJSEAS)-Volume 1.

[3] Broadcast Bus.[online] http://www.ncbi.nlm.nih.gov/pmc/arti cles/PMC4053904/ [May 2014].

[4] Quoc T., Kim M., Lee H. and Eom k., "Wireless Sensor Network apply for the Blind U-bus System," International Journal of u- and eService, Science and Technology Vol. 3, No. 3, September, 2010.

[5] Myat K Khine, Thiri Thandar Aung,"RFID- based Audio Guidance Cane For Blind and Visually Impaired Person, International Journal of Engineering Research \& Technology(IJERT), Vol. 3 Issue 8, August -2014. R. Nicole, "Title of paper with only first word capitalized", J. Name Stand. Abbrev., in press.

[6] New York Transportation Statistics. Available from: $\mathrm{http} / /$ transportation-modes-city.findthedata.org/q /1447/1033/Howmany -people-use-public-transportation-tocommute-in-New-YorkNew-York. Accessed 25 Novmber.

[7] Talking signs Inc.2000. [online] http://www.ski.org/Rehab/W Crandall/introts.html [June 2007].

[8] Miesenberger, K. [et al.] [eds.] Computers Helping People with Special Needs, LNCS, vol. 4061 Springer Berlin / Heidelberg, 10th International Conference, ICCHP 2006, Linz, Austria, July 11-13, 2006.

[9] Hersh, M.A., Johnson, M.A. Assistive Technology for Visually Impaired and Blind People, Springer, 2008.

[10] Crudden A., McBroom L.W. Barriers to employment: a survey of employed persons who are visually impaired. Journal of Visual Impairment and Blindness. 1999;93:341350 . 